\title{
The biomechanical model table tennis ball smash mechanics characteristics
}

\author{
Shaowen Yan ${ }^{1}$, Kunling Qin², Bing Zhang ${ }^{1, a}$ \\ ${ }^{1}$ Institute of Physical Education, Huanggang Normal University, Huangzhou 438000, China \\ ${ }^{2}$ Yidu Gaobazhou Middle School, Yidu 443300, China
}

\begin{abstract}
Table tennis smash model is established according to the principle of geometry, depending on the high ball and low shot the ball trajectory diagram and shot point location, through the establishment of though laser equation, this paper based on constrained particle dynamics equation, get the torque on the shoulder and elbow. According to the shot, according to different net surface vertical distance for table tennis landing point of difference, six different with the Angle of the ball low through a network under the location data. When the drop shot, should try to be arms straight ahead and keep the vertical ball, athletes swing elevation, and the greater the chance of the ball through the net.
\end{abstract}

\section{Introduction}

Table tennis movement is a grid type of competition sports, and random service mode is not affected by the other party, at the same time has also been a lot of research of experts. At the same time, the free service mode is also a powerful way of restraint rivals. Smash belongs to the basic technology of table tennis, also has strong offensive links in the table tennis. To smash the mechanical analysis for the later research on effective competition strategy to lay the foundation [1-3]. Angle is not a normal incidence, the area and the ball is not perpendicular to each other, in general, players when they hit the ball hit the ball is a certain Angle, and inclined to shoot the ball into the other site area, in this paper, according to this phenomenon.

\section{Model establishments}

\subsection{Geometric model establishment}

According to geometric principle, it establishes table tennis spiking model, according to high hitting and low hitting differences, it gets ball trajectory graph and hitting drop point position.

When athlete spikes, he should try to manage to stretch arms to right ahead and remain vertical to hitting point, athlete take-off height gets higher, and ball over net probability would be larger; with ball located hitting point height differences, presented over net trajectory and landing point are also different, therefore it gets long ball and short ball; according to hitting point vertical heights differences, the paper classifies six different heights phases that are respectively. And it gets Table 1 according to hitting point to net vertical distance differences $[4,5]$.

By Table 1, it is clear that athlete hitting points to net surface distances are different, table tennis drop points also have differences, and athlete hitting heights differences can let positions that ball over net to be different.

Table 1. Ball flies across lowest net point's landing point data indication.

\begin{tabular}{|c|c|c|c|c|c|c|}
\hline $\begin{array}{c}\text { Vertical } \\
\text { height/H }\end{array}$ & 0.6 & 0.7 & 0.8 & 0.9 & 1.0 & 1.1 \\
\hline $\begin{array}{c}\text { Hitting point } \\
\text { and net distance } \\
0.75 \mathrm{~m}\end{array}$ & 7.23 & 6.07 & 4.15 & 3.65 & 2.74 & 1.12 \\
\hline $\begin{array}{c}\text { Hitting point } \\
\text { and net distance } \\
0.5 \mathrm{~m}\end{array}$ & 5.55 & 4.05 & 3.10 & 3.01 & 2.49 & 1.08 \\
\hline $\begin{array}{c}\text { Hitting point } \\
\text { and net distance } \\
0.25 \mathrm{~m}\end{array}$ & 4.78 & 3.02 & 2.05 & 1.55 & 1.25 & 1.04 \\
\hline
\end{tabular}

2.2 Low dropping ball's spiking trajectory and hitting drop point improvement under geometric model

Similarly, as Figure 1 show, the paper according to hitting point vertical heights differences, it classifies six different heights phases that are respectively $(0.6,0.7,0.8$,

\footnotetext{
a Corresponding author: tiyuxi@qq.com
} 
$0.9,1.0,1.1)$, and according to hitting point to net vertical distances differences and get Table 2 .

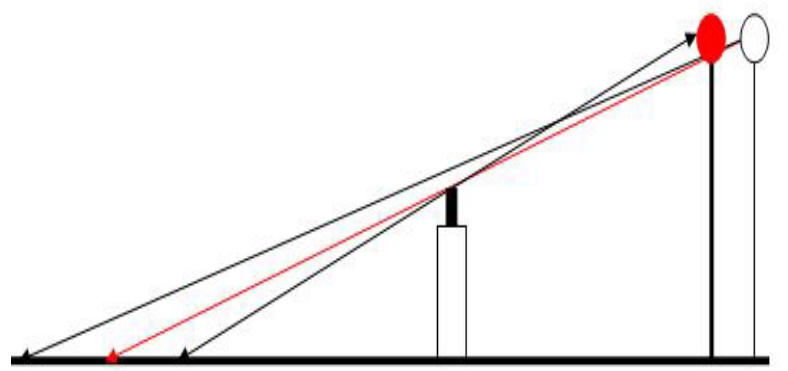

Figure 1. Spiking trajectory and drop points.

Table 2. After shortening hitting point to vertical net $0.1 \mathrm{~m}$ ball flying across lowest net point landing point data indication.

\begin{tabular}{|c|c|c|c|c|c|c|}
\hline Vertical height/H & 0.6 & 0.7 & 0.8 & 0.9 & 1.0 & 1.1 \\
\hline $\begin{array}{c}\text { Hitting point and } \\
\text { net distance } \\
0.75 \mathrm{~m}\end{array}$ & 10.16 & 7.01 & 6.73 & 5.45 & 4.6 & 3.15 \\
\hline $\begin{array}{c}\text { Hitting point and } \\
\text { net distance } \\
0.5 \mathrm{~m}\end{array}$ & 6.32 & 3.89 & 2.68 & 1.48 & 0.98 & 0.87 \\
\hline $\begin{array}{c}\text { Hitting point and } \\
\text { net distance } \\
0.25 \mathrm{~m}\end{array}$ & 5.66 & 4.49 & 3.09 & 2.15 & 1.48 & 1.08 \\
\hline
\end{tabular}

By Table 2 and Figure 1, it is clear that shorten hitting point to net vertical distance $0.1 \mathrm{~m}$, which can clearly get that with respect to low hitting, though only shorten hitting point to net $0.1 \mathrm{~m}$ vertical distance, ball hitting drop point positions have great differences. Hitting drop point in opponent area occurs to great changes.

\subsection{Change hitting angle}

Spiking release angle is not in $90^{\circ}$ vertical incidence, and ball also not vertical enters into opponent field, generally speaking, when athlete hitting, it will hit at certain angles, and oblique hit ball down to opponent field.

Set hitting point and field edge vertical distance is $0.5 \mathrm{~m}$, when athlete hits, deflects rightward $45^{\circ}$ to release and hit, and get hitting point to drop point distance $S$ is:

$$
S=\frac{0.5}{\cos 45^{\circ}}=\frac{0.5}{\sqrt{2} / 2}=0.7072 \mathrm{~m}
$$

Similarly when deflection angle is $30^{\circ}$, it gets hitting point to dropping point vertical projection distance $S$ is:

$$
S=\frac{0.5}{\cos 30}=\frac{0.5}{\sqrt{3} / 2}=0.574 m
$$

\subsection{Hitting instant arms rotational inertia calculation}

The paper establishes Lagrange equation [6], the paper gets restricted particle dynamical equation, from which
Lagrange function $L$ is difference between system kinetic energy $K$ and potential energy $P: L=K-P$ System dynamical equation is:

$$
F_{i}=\frac{d}{d t}\left(\frac{\partial L}{\partial q_{i}}-\frac{\partial L}{\partial q_{i}}\right) \quad i=1,2, \mathrm{~L}, n
$$

In above formula $\stackrel{\&}{\underset{q}{q}}$ is corresponding speed, $q_{i}$ is dynamic energy and potential energy coordinate, $F_{i}$ is the $i$ coordinate acting force, thigh and shank included angles with coordinate axis are respectively $\theta_{1}, \theta_{2}$, lengths are respectively $l_{1}, l_{2}$, Arms front part and arms post part gravity center position distances with elbow joint center and knee joint are respectively $p_{1}, p_{2}$, therefore it is clear that arms gravity center coordinate $\left(X_{1}, Y_{1}\right)$ is:

$$
\left\{\begin{array}{l}
X_{1}=p_{1} \sin \theta_{1} \quad Y_{1}=p_{1} \cos \theta_{1} \\
X_{2}=l_{1} \sin \theta_{1}+p_{2} \sin \left(\theta_{1}+\theta_{2}\right) \quad Y_{2}=-l_{1} \cos \theta_{1}-p_{2} \cos \left(\theta_{1}+\theta_{2}\right)
\end{array}\right.
$$

Similarly, arms gravity center coordinate $\left(X_{2}, Y_{2}\right)$ can also be solved. System dynamic energy $E_{k}$ and system potential energy $E_{p}$ expressions are:

$$
\left\{\begin{array}{l}
E_{k}=E_{k 1}+E_{k 2}, E_{k 1}=\frac{1}{2} m_{1} p_{1}^{2} \dot{\theta}_{1}^{2} \\
E_{k 2}=\frac{1}{2} m_{2} l_{1}^{2} \dot{\theta}_{1}^{2}+\frac{1}{2} m_{2} p_{2}^{2}\left(\dot{\theta}_{1}+\dot{\theta}_{2}\right)^{2}+m_{2} l_{2} p_{2}\left(\dot{\theta}_{01}^{2}+\dot{\theta}_{1} \dot{\theta}_{2}\right) \cos \theta_{2} \\
E_{p}=E_{p 1}+E_{p 2}, E_{p 1}=\frac{1}{2} m_{1} g p_{1}\left(1-\cos \theta_{1}\right) \\
E_{p 2}=m_{2} g p_{2}\left[1-\cos \left(\theta_{1}+\theta_{2}\right)\right]+m_{2} g l_{1}\left(1-\cos \theta_{1}\right)
\end{array}\right.
$$

Write above formula into Lagrange function expression, by Lagrange system dynamical equation, it can get hip joint and knee joint moments of force $M_{h}$ and $M_{k}$ as:

$$
\begin{aligned}
{\left[\begin{array}{c}
M_{h} \\
M_{k}
\end{array}\right]=} & {\left[\begin{array}{ll}
D_{11} & D_{12} \\
D_{21} & D_{22}
\end{array}\right]\left[\begin{array}{l}
\ddot{\theta}_{1} \\
\ddot{\theta}_{2}
\end{array}\right]+\left[\begin{array}{ll}
D_{111} & D_{122} \\
D_{211} & D_{222}
\end{array}\right]\left[\begin{array}{c}
\dot{\theta}_{1}^{2} \\
\dot{\theta}_{2}^{2}
\end{array}\right]+} \\
& {\left[\begin{array}{ll}
D_{112} & D_{121} \\
D_{212} & D_{221}
\end{array}\right]\left[\begin{array}{c}
\dot{\theta}_{1} \dot{\theta}_{2} \\
\dot{\theta}_{2} \dot{\theta}_{1}
\end{array}\right]+\left[\begin{array}{c}
D_{1} \\
D_{2}
\end{array}\right] }
\end{aligned}
$$

In above formula, $D_{i j k}$ is as following result:

$$
\begin{gathered}
D_{111}=0 \quad D_{222}=0 \quad D_{121}=0 \\
D_{22}=m_{2} p_{2}^{2} \\
D_{11}=m_{1} p_{1}^{2}+m_{2} p_{2}^{2}+m_{2} l_{1}^{2}+2 m_{2} l_{1} p_{2} \cos \theta_{2} \\
D_{12}=m_{2} p_{2}^{2}+m_{2} l_{1} p_{2} \cos \theta_{2} \quad D_{21}=m_{2} p_{2}^{2}+m_{1} l_{1} p_{2} \cos \theta_{2}
\end{gathered}
$$




$$
\begin{gathered}
D_{1}=\left(m_{1} p_{1}+m_{2} l_{1}\right) g \sin \theta_{1}+m_{2} p_{2} g \sin \left(\theta_{1}+\theta_{2}\right) \\
D_{122}=-m_{2} l_{1} p_{2} \sin \theta_{2} \\
D_{211}=m_{2} l_{1} p_{2} \sin \theta_{2} \\
D_{112}=-2 m_{2} l_{1} p_{2} \sin \theta_{2} \\
D_{212}=D_{122}+D_{211} \\
D_{2}=m_{2} p_{2} g \sin \left(\theta_{1}+\theta_{2}\right)
\end{gathered}
$$

Combine with theoretical equation, analyze when table tennis players spike, hand joint mechanical movement combines with shoulder joint, elbow joint mechanical analyses to research on table tennis spiking technique.

\subsection{Establish moment of momentum theorem model}

When apply mechanical conservation law into solving problems, at first it should select reasonable research objects, and make correct force analysis of researched objects, the next is on the basis of force analysis, refer to conservation law to check problems, and finally according to conservation law, establish equation and solve problems.

Set $I$ is one rigid body rotational inertia, suffered torque $M$ effects, from which angular accelerated speed $\beta$ is constant, the rigid body at time $t_{1}$ angular speed is $\omega_{1}$, the rigid body at time $t_{2}$ angular speed is $\omega_{2}$, and get: $M=I \beta=I \frac{\omega_{2}-\omega_{1}}{t_{2}-t_{1}}$

Transform and get: $M\left(t_{2}-t_{1}\right)=I\left(\omega_{2}-\omega_{1}\right)$

When $M=M(t)$, it has: $M(t)\left(t_{2}-t_{1}\right)=I\left(\omega_{2}-\omega_{1}\right)$

It gets moment of momentum formula, from which $M\left(t_{2}-t_{1}\right)$ is impulsive moment, $I \omega$ is moment of momentum, from formula, it is clear that rigid body impulsive moment variable quantity and moment of momentum variable quantity are equal.

In moment of momentum theorem, time and torque product is equal to impulsive moment that represents object rotational accumulation effect under external force moment influences. Angular speed and rotational inertia product is rigid body state when rotating. With external force moment increases and acting time enlarges, rigid body rotational state changes are increasing accordingly.

When human body moves, human body generated rotational inertial is changing, due to rotational variables changes, different times rotational inertias are different, set $t_{1}$ time rotational inertia is $I_{1}, t_{2}$ time rotational inertia is $I_{2}$, therefore, above formula can be revised into: $\left.M(t)\left(t_{2}-t_{1}\right)=I_{2} \omega_{2}-I_{1} \omega_{1}\right)$
For Human body basic movement rules, it should meet: $I \omega=0, \quad \sum M \square t=0$

Now it enters into soaring phase, assume human body meets: $I_{1} \omega_{1}+I_{2} \omega_{2}=0$

Besides, it should also meet human body surround $I_{1} \omega_{1}$ to rotate, then the kind of movement form is lengthwise relative movement, in spiking process, solve the sum of human body moment of momentum vectors is 0 , according to correlation law, we get that human body will suffer ball acted a reaction force that let people produce moment of momentum, so that reduce spiking process strength sizes and it is bad for spiking stability, but if in the spiking process, due to body each part suffered active force effects, which causes rotational inertia increase, it will further produce an advancing moment of momentum effects; according to energy conservation law, we know that human body also will produce a reverse active force effects at this time, so that let human body move relative to ball, based on which it increases arms swinging distance and concentrates on whole body strength to hit the ball.

Hand air angular speed changes, in case moment of momentum remains unchanged, rotational inertial will reduce with angular speed increases, when athlete prepares for swinging racket, athlete himself can further control rotational angular speed by changing self-rotational inertia.

Athlete take-off legs slightly bend and let gravity center and body rotational axis come to terms and reduce so that can reduce rotational inertia, and then it further achieves the efficiency of increasing rotational angular speed, when athlete takes off and arrives at top point, athlete should try to adjust body stability and let rotational angular speed reduce as much as possible [7], at this time, athlete should lift two legs backward, and let gravity center to be far away from rotational axis. Then arrive at stable contacting ball state.

\section{Conclusions}

Smash belongs to the basic technology of table tennis, also has strong offensive links in the table tennis. To smash the mechanical analysis for the later research on effective competition strategy to lay the foundation. Through the establishment of the geometric model, when athletes spiking, should try to be arms straight ahead and keep the vertical ball, athletes swing elevation, and the greater the chance of the ball through the net, the ball was located by the height of the ball is different, after the net of trajectory and landing site is different also, therefore, in this paper, the height of the six different period of analysis. According to the athlete spiking Angle is not a normal incidence, and the ball is not perpendicular to each other, especial phenomena, research in LouQiuShi Angle shots, ball diagonal shot down to the position of each district is different. 


\section{References}

1. Y. J. Zhong, D. Z. Wang and J. Wang, Journal of Beijing Sport University, 31, 456-459(2008).

2. H. Yang and Z. M. Guan, Computer Simulation, 28, 230-233(2011).

3. Z. Sun, G. X. Yu, M. Guo, L. L. Zhu, J. Yang and Z. B. He, China Sport Science, 28, 69-71(2008).

4. Q. F. Zhang and J. Su, China Sport Science and Technology, 41, 90-92(2005).
5. C. C. Zhang, L. Lin and H. Q. Hu, 18, 123-124(2010).

6. A. Escobar, V. Negro, J. S. Lopez-Gutierrez et al. Journal of Coastal Research, 841-846(2016).

7. F. M. Zhang, and Z. L. Liu, S. Kemal, Abstracts of papers of the American Chemical Society, 240, 71-CARB(2010). 